\title{
AN APPLICATION OF THE BACKBONE DECOMPOSITION TO SUPERCRITICAL SUPER-BROWNIAN MOTION WITH A BARRIER
}

\author{
A. E. KYPRIANOU,* University of Bath \\ A. MURILLO-SALAS, ${ }^{* *}$ Universidad de Guanajuato \\ J. L. PÉREZ,*** Mexico Autonomous Institute of Technology
}

\begin{abstract}
We analyse the behaviour of supercritical super-Brownian motion with a barrier through the pathwise backbone embedding of Berestycki, Kyprianou and Murillo-Salas (2011). In particular, by considering existing results for branching Brownian motion due to Harris and Kyprianou (2006) and Maillard (2011), we obtain, with relative ease, conclusions regarding the growth in the right-most point in the support, analytical properties of the associated one-sided Fisher-Kolmogorov-Petrovskii-Piscounov wave equation, as well as the distribution of mass on the exit measure associated with the barrier.

Keywords: Super-Brownian motion; backbone decomposition; killed super-Brownian motion

2010 Mathematics Subject Classification: Primary 60J68; 35C07
\end{abstract}

\section{Introduction}

Suppose that $X=\left\{X_{t}: t \geq 0\right\}$ is a (one-dimensional) superdiffusion with motion corresponding to that of a Brownian motion with drift $-\rho \in \mathbb{R}$, stopped at 0 , and branching mechanism $\psi$ taking the form

$$
\psi(\lambda)=-\alpha \lambda+\beta \lambda^{2}+\int_{(0, \infty)}\left(\mathrm{e}^{-\lambda x}-1+\lambda x\right) \Pi(\mathrm{d} x)
$$

for $\lambda \geq 0$, where $\alpha=-\psi^{\prime}\left(0^{+}\right) \in(0, \infty), \beta \geq 0$, and $\Pi$ is a measure concentrated on $(0, \infty)$ which satisfies $\int_{(0, \infty)}\left(x \wedge x^{2}\right) \Pi(\mathrm{d} x)<\infty$. We also insist that $\beta>0$ if $\Pi \equiv 0$. The existence of this class of superprocesses is guaranteed by [4], [6], and [8].

Let $\mathcal{M}_{F}(I)$ be the space of finite measures on $I \subseteq \mathbb{R}$, and note that $X$ is an $\mathcal{M}_{F}[0, \infty)$ valued Markov process under $\mathbb{P}_{\mu}$ for each $\mu \in \mathcal{M}_{F}[0, \infty)$, where $\mathbb{P}_{\mu}$ is law of $X$ with initial configuration $\mu$. One may think of $\mathbb{P}_{\mu}$ as a law on the càdlàg path space $\mathcal{X}:=D([0, \infty) \times$ $\left.\mathcal{M}_{F}[0, \infty)\right)$. Henceforth we shall use standard inner product notation: for $f \in C_{b}^{+}[0, \infty)$ and $\mu \in \mathcal{M}_{F}[0, \infty)$,

$$
\langle f, \mu\rangle=\int_{\mathbb{R}} f(x) \mu(\mathrm{d} x) .
$$

Accordingly, we shall write $\|\mu\|=\langle 1, \mu\rangle$.

Received 20 October 2011; revision received 2 February 2012.

* Postal address: Department of Mathematical Sciences, University of Bath, Claverton Down, Bath, BA2 7AY, UK.

** Postal address: Departamento de Matemáticas, Universidad de Guanajuato, Jalisco s/n, Mineral de Valenciana, Guanajuato, Gto. CP 36240, México.

*** Postal address: Department of Statistics, Mexico Autonomous Institute of Technology, Rio Hondo 1, Tizapan 1 San Angel, Mexico DF 01000, Mexico. 
Recall that the total mass of the process $X$ is a continuous-state branching process with branching mechanism $\psi$. Since there is no interaction between spatial motion and branching, we can characterise our $\psi$-superdiffusion as supercritical on account of the assumption that $\alpha=-\psi^{\prime}\left(0^{+}\right)>0$. Such processes may exhibit explosive behaviour; however, under the conditions assumed above, $X$ remains finite at all positive times. We insist moreover that $\psi(\infty)=\infty$, which means that, with positive probability, the event $\lim _{t \uparrow \infty}\left\|X_{t}\right\|=0$ will occur. Equivalently, this means that the total mass process does not have monotone increasing paths; see, for example, the summary in Chapter 10 of [17]. The probability of the event

$$
\mathcal{E}:=\left\{\lim _{t \uparrow \infty}\left\|X_{t}\right\|=0\right\}
$$

is described in terms of the largest root, say $\lambda^{*}$, of the equation $\psi(\lambda)=0$. It is known (cf. Chapter 8 of [17]) that $\psi$ is strictly convex with $\psi(0)=0$ and, hence, since $\psi(\infty)=\infty$ and $\psi^{\prime}\left(0^{+}\right)<0$, it follows that there are exactly two roots in $[0, \infty)$, one of which is always 0 . For $\mu \in \mathcal{M}_{F}[0, \infty)$, we have

$$
\mathbb{P}_{\mu}\left(\lim _{t \uparrow \infty}\left\|X_{t}\right\|=0\right)=\mathrm{e}^{-\lambda^{*}\|\mu\|}
$$

It is a straightforward exercise (cf. Lemma 2 of [3] or Theorem 2.6 of [25]) to show that the law of $X$ under $\mathbb{P}_{\mu}$ conditioned on $\&$ is that of another superdiffusion with the same motion component as $X$, but with a new branching mechanism which is given by $\psi^{*}(\lambda)=\psi\left(\lambda+\lambda^{*}\right)$ for $\lambda \geq 0$. Said another way, the aforementioned superdiffusion has a semigroup characterised by the nonlinear equation (4) below with the quantity $\psi$ replaced by $\psi^{*}$. We denote its law by $\mathbb{P}_{\mu}^{*}$.

In this paper we shall also assume that

$$
\int^{\infty} \frac{1}{\sqrt{\int_{\lambda^{*}}^{\xi} \psi(u) \mathrm{d} u}} \mathrm{~d} \xi<\infty .
$$

Condition (1) implies in particular that $\int^{\infty} 1 / \psi(\xi) \mathrm{d} \xi<\infty$ (cf. [25]), which in turn guarantees that the event $\mathcal{E}$ agrees with the event of extinction, namely, $\left\{\zeta^{X}<\infty\right\}$, where

$$
\zeta^{X}=\inf \left\{t>0:\left\|X_{t}\right\|=0\right\}
$$

Note that (1) cannot be satisfied for branching mechanisms which are the Laplace exponents of bounded variation, spectrally positive Lévy processes.

In this paper our objective is to show the robustness of a recent pathwise backbone decomposition, described in detail in the next section, as a mechanism for transferring results from branching diffusions directly into the setting of superprocesses. We shall do this by demonstrating how two related fundamental results for branching Brownian motion with a killing barrier induce the same results for a $\psi$-super-Brownian motion with killing at the origin. The latter, which we shall denote by $X^{+}=\left\{X_{t}^{+}: t \geq 0\right\}$, can be defined on the same probability space as $X$ by simply taking

$$
X_{t}^{+}(\cdot)=X_{t}(\cdot \cap(0, \infty)) .
$$

For $f \in C_{b}^{+}(0, \infty), \mu \in \mathcal{M}_{F}(0, \infty), x>0$, and $t \geq 0$,

$$
-\log \mathbb{E}_{\mu}\left(\mathrm{e}^{-\left\langle f, X_{t}\right\rangle}\right)=\int_{(0, \infty)} u_{f}(x, t) \mu(\mathrm{d} x), \quad t \geq 0,
$$


describes the semigroup of $X$, where $u_{f}$ is the unique positive solution to

$$
u_{f}(x, t)=\mathrm{E}_{x}^{-\rho}\left[f\left(B_{t \wedge \tau_{0}}\right)\right]-\mathrm{E}_{x}^{-\rho}\left[\int_{0}^{t \wedge \tau_{0}} \psi\left(u_{f}\left(B_{s}, t-s\right)\right) \mathrm{d} s\right], \quad x, t \geq 0 .
$$

Here, $\mathrm{E}_{x}^{-\rho}$ is the expectation with respect to $\mathrm{P}_{x}^{-\rho}$, under which $\left\{B_{t}: t \geq 0\right\}$ is a Brownian motion with drift $-\rho$, issued from $x \geq 0$, and $\tau_{0}=\inf \left\{t>0: B_{t}<0\right\}$. The reader is referred to Theorem 1.1 of [5], Proposition 2.3 of [14], and Proposition 2.2 of [27] for further details; see also [6] and [8] for a general overview.

Our first result, based on the branching particle analogue in [15], shows that the classical growth of the right-most point in the support and its intimate relation with nonnegative stationary solutions to (4) can also be seen in the superprocess context. Specifically, we mean solutions of the form $u(x, t)=\Phi(x)$, which necessarily solve

$$
\Phi(x)=\mathrm{E}_{x}^{-\rho}\left[\Phi\left(B_{t \wedge \tau_{0}}\right)\right]-\mathrm{E}_{x}^{-\rho}\left[\int_{0}^{t \wedge \tau_{0}} \psi\left(\Phi\left(B_{S}\right)\right) \mathrm{d} s\right], \quad x \geq 0 .
$$

If we additionally suppose, for technical reasons which will soon become apparent, that $\Phi$ monotonically connects 0 at the origin to $\lambda^{*}$ at $+\infty$, then it is a straightforward exercise using the classical Feynman-Kac representation of solutions to ordinary differential equations to show that $\Phi$ solves the differential equation

$$
\frac{1}{2} \Phi^{\prime \prime}(x)-\rho \Phi^{\prime}(x)-\psi(\Phi(x))=0 \quad \text { on } x>0 \text { with } \Phi(0+)=0 \text { and } \Phi(+\infty)=\lambda^{*} .
$$

In this case we call $\Phi$ a wave solution to (5).

Theorem 1. (Strong law for the support.) Define

$$
R_{t}^{X}:=\inf \left\{y>0: X_{t}(y, \infty)=0\right\}=\inf \left\{y>0: X_{t}^{+}(y, \infty)=0\right\},
$$

and denote the extinction time of $X^{+}$by

$$
\zeta_{+}^{X}=\inf \left\{t>0:\left\|X_{t}^{+}\right\|=0\right\} .
$$

(i) Assume that $-\infty<\rho<\sqrt{2 \alpha}$. Then, for all $x>0$,

$$
\lim _{t \rightarrow \infty} \frac{R_{t}^{X}}{t}=\sqrt{2 \alpha}-\rho \quad \text { on }\left\{\zeta_{+}^{X}=\infty\right\}
$$

$\mathbb{P}_{\delta_{x}}$-almost surely, and

$$
\Phi(x):=-\log \mathbb{P}_{\delta_{x}}\left(\zeta_{+}^{X}<\infty\right) \text { for all } x>0
$$

is the unique wave solution to (5).

(ii) For all $\rho \geq \sqrt{2 \alpha}$, there exists no monotone wave solution to (5) and

$$
\mathbb{P}_{\delta_{x}}\left(\zeta_{+}^{X}<\infty\right)=1 \text { for all } x>0 .
$$

Remark 1. Whilst Theorem 1 offers results on the existence and uniqueness of solutions to (5), we do not claim that these are necessarily new. Indeed, we may extract the same or similar results using the methods in, for example, [16], [22], and [26]. See also the discussion in Remark 2 below. 
Our second result looks at the distribution of mass that is absorbed at the origin, when $\rho$ takes the critical value $\sqrt{2 \alpha}$, in the spirit of recent results of Addario-Berry and Broutin [1], Aïdékon et al. [2], and Maillard [20]. In order to describe this result, we need to introduce the concept of Dynkin's exit measures.

For each $x \in \mathbb{R}$, suppose that we defined the superprocess $Y=\left\{Y_{t}: t \geq 0\right\}$ under $\mathbb{Q}_{\delta_{x}}$ to have the same branching mechanism as $\left(X, \mathbb{P}_{\delta_{x}}\right)$; however, the underlying motion associated with $Y$ is that of a Brownian motion with drift $-\rho$ (i.e. no stopping at 0$)$. The existence of $\left(Y, \mathbb{Q}_{\delta_{x}}\right)$ is justified through the same means as for $\left(X, \mathbb{P}_{\delta_{x}}\right)$. In principle, it is possible to construct these two processes on the same probability space; however, this is unnecessary for our purposes. For each $z, t \geq 0$, define the space-time domain $D_{-z}^{t}=\{(x, u) \in \mathbb{R} \times[0, \infty): u<t$, $x>-z$. According to Dynkin's theory of exit measures outlined in Section 7 of [7] and Section 1 of [9], it is possible to describe the mass in the superprocess $Y$ as it first exits the domain $D_{-z}^{t}$. In particular, according to the characterisation for branching Markov exit measures given in Section 1.1 of [9], the random measure $Y_{D_{-z}^{t}}$ is supported on $\partial D_{-z}^{t}=$ $(\{-z\} \times[0, t)) \cup([-z, \infty) \times\{t\})$ and is characterised by the Laplace functional

$$
\mathbb{Q}_{\delta_{x}}\left(\exp \left\{-\left\langle f, Y_{D_{-z}^{t}}\right\rangle\right\}\right)=\mathrm{e}^{-u_{f}^{z}(x, t)},
$$

where $x \geq-z, f \in C_{b}([-z, \infty) \times[0, \infty))$, and $u_{f}^{z}(x, t)$ uniquely solves, amongst nonnegative solutions (cf. Theorem 6.1 of [7]), the equation

$$
\begin{aligned}
u_{f}^{z}(x, t)= & \mathrm{E}_{x}^{-\rho}\left[f\left(B_{t \wedge \tau_{-z}}, t \wedge \tau_{-z}\right)\right] \\
& -\mathrm{E}_{x}^{-\rho}\left[\int_{0}^{t \wedge \tau_{-z}} \psi\left(u_{f}^{z}\left(B_{u}, t-u\right)\right) \mathrm{d} u\right], \quad x \geq-z, t \geq 0,
\end{aligned}
$$

where $\tau_{-z}=\inf \left\{t>0: B_{t}<-z\right\}$. Intuitively speaking, we should think of $Y_{D_{-z}^{t}}$ as the analogue of the atomic measure supported on $\partial D_{-z}^{t}$ which describes the collection of particles and their space-time positions in a branching Brownian motion with drift $-\rho$ that are first in their genealogical line of descent to exit the space-time domain $(-z, \infty) \times[0, t)$.

In the case that $\rho \geq \sqrt{2 \alpha}$, it was shown in Theorem 3.1 of [18] that the limiting random measure $Y_{D_{-z}}=\lim _{t \uparrow \infty} Y_{D_{-z}^{t}}$ (which exists almost surely by monotonicity) is almost surely finite and has total mass which satisfies

$$
\mathbb{Q}_{\delta_{x}}\left(\exp \left\{-\theta\left\|Y_{D_{-z}}\right\|\right\}\right)=\mathrm{e}^{-v_{\theta}(x+z)}
$$

for $\theta \geq 0$ and $x \geq-z$, where

$$
\frac{1}{2} v_{\theta}^{\prime \prime}(x)-\rho v_{\theta}^{\prime}(x)-\psi\left(v_{\theta}(x)\right)=0,
$$

with $v_{\theta}(0)=\theta$. In particular, $\left\{v_{\theta}(x): x \geq 0\right\}$ is the semigroup of a continuous-state branching process whose branching mechanism satisfies

$$
\psi_{D}(\lambda)=\Psi^{\prime}\left(\Psi^{-1}(\lambda)\right)
$$

for $\lambda \in\left[0, \lambda^{*}\right]$, where $\Psi$ is the unique monotone solution to the wave equation

$$
\frac{1}{2} \Psi^{\prime \prime}(x)+\rho \Psi^{\prime}(x)-\psi(\Psi(x))=0 \quad \text { on } \mathbb{R} \text { with } \Psi(-\infty)=\lambda^{*} \text { and } \Psi(+\infty)=0 .
$$

Indeed, it was shown in Theorem 3.1 of [18] that $\left\|Y_{D}\right\|:=\left\{\left\|Y_{D_{-z}}\right\|: z \geq 0\right\}$ is a continuous-state branching process with growth rate $\rho+\sqrt{\rho^{2}-2 \alpha}$. 
We are now ready to state our second main result, based on the branching Brownian motion analogue in [20], which in particular focuses on the case that the underlying motion has a critical speed $\sqrt{2 \alpha}$.

Theorem 2. (Absorbed mass at criticality.) Set $\rho=\sqrt{2 \alpha}$. Assume that, for some $\varepsilon>0$,

$$
\int_{[1, \infty)} x(\log x)^{2+\varepsilon} \Pi(\mathrm{d} x)<\infty
$$

Then, for each $z, x>0$, we have

$$
\mathbb{Q}_{\delta_{x}}\left(\left\|Y_{D_{-z}}\right\|>t\right) \sim \sqrt{2 \alpha} \frac{(x+z) \mathrm{e}^{(x+z) \sqrt{2 \alpha}}}{t(\log t)^{2}}
$$

as $t \uparrow \infty$.

Note that, in terms of our earlier notation, we see that $X_{t}^{+}$under $\mathbb{P}_{\delta_{x}}$ has the same law as $\left.Y_{D_{0}^{t}}\right|_{(0, \infty) \times\{t\}}$ under $\mathbb{Q}_{\delta_{x}}$. Whilst Theorem 1 therefore concerns the spatial evolution of the support of the measure $Y_{D_{0}^{t}}$ away from the origin for speeds $\rho>\sqrt{2 \alpha}$, by contrast, Theorem 2 addresses the distribution of mass accumulated at the origin by the same measure, at the critical speed $\sqrt{2 \alpha}$.

The remainder of this paper is structured as follows. In Section 2 we give a brief overview of the backbone decomposition for $X$, noting that similar decompositions also hold for a number of other processes used in this paper. In Section 3 we prove Theorem 1, and in Section 4 we prove Theorem 2.

\section{The backbone decomposition and Poissonisation}

As alluded to above, our results are largely driven by the backbone decomposition, recently described in the pathwise sense by Berestycki et al. [3] for conservative processes. Note that backbone decompositions have been known in the earlier and more analytical setting of semigroup decompositions through the work of Evans and O'Connell [12] and Engländer and Pinsky [11], as well as in the pathwise setting in the work of Salisbury and Verzani [23], [24].

To describe the backbone decomposition in detail, consider the process $\left\{\Lambda_{t}^{X}: t \geq 0\right\}$ which has the following pathwise construction. First sample from a branching particle diffusion with branching generator

$$
F(r)=q\left(\sum_{n \geq 0} p_{n} r^{n}-r\right)=\frac{1}{\lambda^{*}} \psi\left(\lambda^{*}(1-r)\right), \quad r \in[0,1],
$$

and particle motion which is that of a Brownian motion with drift $-\rho$, stopped at the origin. Note that in the above generator, $q$ is the rate at which individuals reproduce and $\left\{p_{n}: n \geq 0\right\}$ is the offspring distribution. With the particular branching generator given by $(8), q=\psi^{\prime}\left(\lambda^{*}\right)$, $p_{0}=p_{1}=0$, and, for $n \geq 2, p_{n}:=p_{n}[0, \infty)$, where, for $y \geq 0$, we defined the measure $p_{n}(\cdot)$ on $\{2,3,4, \ldots\} \times[0, \infty)$ by

$$
p_{n}(\mathrm{~d} y)=\frac{1}{\lambda^{*} \psi^{\prime}\left(\lambda^{*}\right)}\left\{\beta\left(\lambda^{*}\right)^{2} \delta_{0}(\mathrm{~d} y) \mathbf{1}_{\{n=2\}}+\left(\lambda^{*}\right)^{n} \frac{y^{n}}{n !} \mathrm{e}^{-\lambda^{*} y} \Pi(\mathrm{d} y)\right\} .
$$

If we denote the aforesaid branching particle diffusion by $Z^{X}=\left\{Z_{t}^{X}: t \geq 0\right\}$ then we shall also insist that the configuration of particles in space at time $0, Z_{0}$, is given by an independent Poisson 
random measure with intensity $\lambda^{*} \mu$. Next, dress the branches of the spatial tree that describes the trajectory of $Z^{X}$ in such a way that a particle at the space-time position $(\xi, t) \in[0, \infty)^{2}$ has an independent $\mathcal{X}$-valued trajectory grafted on to it with rate

$$
2 \beta \mathrm{dN}_{\xi}^{*}+\int_{0}^{\infty} y \mathrm{e}^{-\lambda^{*} y} \Pi(\mathrm{d} y) \mathrm{d} \mathbb{P}_{\xi \delta_{y}}^{*} .
$$

Here the measure $\mathbb{N}_{\xi}^{*}$ is the excursion measure (cf. [9], [10], and [19]) on the space $\mathcal{X}$ which satisfies

$$
\mathbb{N}_{x}^{*}\left(1-\mathrm{e}^{-\left\langle f, X_{t}\right\rangle}\right)=u_{f}^{*}(x, t)
$$

for $x, t \geq 0$ and $f \in C_{b}^{+}[0, \infty)$, where $u_{f}^{*}(x, t)$ is the unique solution to (4) with the branching mechanism $\psi$ replaced by $\psi^{*}$. Moreover, on the event that an individual in $Z^{X}$ dies and branches into $n \geq 2$ offspring at spatial position $\xi \in[0, \infty)$, with probability $p_{n}(\mathrm{~d} y) \mathbb{P}_{y \delta_{\xi}}^{*}$, an additional independent $\mathcal{X}$-valued trajectory is grafted on to the space-time branching point. The quantity $\Lambda_{t}^{X}$ is now understood to be the total dressed mass present at time $t$ together with the mass present at time $t$ of an independent copy of $\left(X, \mathbb{P}_{\mu}^{*}\right)$ issued at time 0 . We denote the law of $\left(\Lambda^{X}, Z^{X}\right)$ by $\mathbf{P}_{\mu}$.

The backbone decomposition is now summarised by the following theorem lifted from [3].

Theorem 3. For any $\mu \in \mathcal{M}_{F}[0, \infty)$, the process $\left(\Lambda^{X}, \mathbf{P}_{\mu}\right)$ is Markovian and has the same law as $\left(X, \mathbb{P}_{\mu}\right)$. Moreover, for each $t \geq 0$, the law of $Z_{t}^{X}$ given $\Lambda_{t}^{X}$ is that of a Poisson random measure with intensity measure $\lambda^{*} \Lambda_{t}^{X}$.

Not much changes in the above account when we replace the role of $X$ by the superprocess $Y$ or indeed the continuous-state branching process $\left\|Y_{D}\right\|$. Specifically, for the case of $Y$, the motion of the backbone, $Z^{Y}$, is that of a Brownian motion with drift $-\rho$ and $\psi$ remains the same. For the case of $\left\|Y_{D}\right\|$, we may consider the motion process to be that of a particle which remains fixed at a point and the branching mechanism $\psi$ is replaced by $\psi_{D}$.

\section{Proof of Theorem 1}

Proof of $(i)$. Using obvious notation in light of (2), and referring to the discussion following Theorem 2, we necessarily have $R_{t}^{X}$ equal in law to $\inf \left\{y>0:\left.Y_{D_{0}^{t}}\right|_{(0, \infty) \times\{t\}}(y, \infty)=0\right\}$, and the latter is $\mathbb{Q}_{\delta_{x}}$-almost surely bounded above by $R_{t}^{Y}$. It is known from Corollary 3.2 of [18] that, under (1), for any $\rho \leq \sqrt{2 \alpha}$,

$$
\lim _{t \rightarrow \infty} \frac{R_{t}^{Y}}{t}=\sqrt{2 \alpha}-\rho
$$

$\mathbb{Q}_{\delta_{x}}$-almost surely on the survival set of $Y$. It follows that, under the same assumptions,

$$
\limsup _{t \rightarrow \infty} \frac{R_{t}^{X}}{t} \leq \sqrt{2 \alpha}-\rho \quad \text { on }\left\{\zeta_{+}^{X}=\infty\right\}
$$

$\mathbb{P}_{\delta_{x}}$-almost surely.

For the lower bound, note that the backbone decomposition allows us to deduce straight away that, again using obvious notation, on $\left\{\zeta_{+}^{\Lambda^{X}}=\infty\right\}, R_{t}^{Z^{X}} \leq R_{t}^{\Lambda^{X}}$ holds $\mathbf{P}_{\delta_{x}}$-almost surely for each $x>0$. The restriction of the process $Z^{X}$ to $(0, \infty)$ can be formally identified as a branching Brownian motion with killing at the origin. In [15] it was shown that a dyadic branching Brownian motion with drift $-\rho$ and killing at the origin which branches at rate $q$ has 
the property that the asymptotic right-most particle speed is equal to $\sqrt{2 q}-\rho$ on survival. In fact, careful inspection of their proof shows that it is straightforward to replace dyadic branching by a random number of offspring with mean $m \in(1, \infty)$. In that case the right-most speed is equal to $\sqrt{2 q(m-1)}-\rho$. Note that, for the process $Z^{X}$, we easily compute from (8) that $q(m-1)=\alpha$. We now have

$$
\liminf _{t \rightarrow \infty} \frac{R_{t}^{\Lambda^{X}}}{t} \geq \lim _{t \rightarrow \infty} \frac{R_{t}^{Z^{X}}}{t}=\sqrt{2 \alpha}-\rho \quad \text { on }\left\{\zeta_{+}^{Z^{X}}=\infty\right\}
$$

$\mathbf{P}_{\delta_{x}}$-almost surely. Let us temporarily assume however that $\left\{\zeta_{+}^{Z^{X}}<\infty\right\}$ agrees with the event $\left\{\zeta_{+}^{\Lambda^{X}}<\infty\right\}$ under $\mathbf{P}_{\delta_{x}}$. Theorem 3 now allows us to conclude from (10) that

$$
\liminf _{t \rightarrow \infty} \frac{R^{X}}{t} \geq \sqrt{2 \alpha}-\rho \quad \text { on }\left\{\zeta_{+}^{X}=\infty\right\}
$$

$\mathbb{P}_{\delta_{x}}$-almost surely.

To complete the proof of part (i), we must therefore show that $\left\{\zeta_{+}^{Z^{X}}<\infty\right\}$ agrees with the event $\left\{\zeta_{+}^{\Lambda^{X}}<\infty\right\}$ under $\mathbf{P}_{\delta_{x}}$ and that their common probabilities give the unique solution to (5). To this end, first note that the backbone decomposition, and in particular the Poisson embedding of $Z^{X}$ in $\Lambda^{X}$, gives $\left\{\zeta_{+}^{\Lambda^{X}}<\infty\right\} \subseteq\left\{\zeta_{+}^{Z^{X}}<\infty\right\}$. Next note that the backbone decomposition also tells us that $\left\{Z_{0}^{X}(0, \infty)=0\right\} \subseteq\left\{\zeta_{+}^{\Lambda^{X}}<\infty\right\}$. If we define the monotone increasing function $\Phi:[0, \infty) \rightarrow[0, \infty)$ by

$$
\mathrm{e}^{-\Phi(x)}=\mathbb{P}_{\delta_{x}}\left(\zeta_{+}^{X}<\infty\right)=\mathbf{P}_{\delta_{x}}\left(\zeta_{+}^{\Lambda^{X}}<\infty\right),
$$

so that in particular $\Phi(0)=0$, then the previous observations tell us that, for $x>0$,

$$
\mathrm{e}^{-\lambda^{*}} \leq \mathrm{e}^{-\Phi(x)} \leq \mathbf{P}_{\delta_{x}}\left(\zeta_{+}^{Z^{X}}<\infty\right)<1
$$

Note that the right-hand side inequality above is strict as all initial particles in $Z^{X}$ may hit the stopping boundary before branching with positive probability. It is a straightforward exercise to show, using the Markov branching property and the fact that $\Phi(0)=0$, that $\Phi$ respects the relation

$$
\mathrm{e}^{-\Phi(x)}=\mathbb{E}_{\delta_{x}}\left(\mathbb{P}_{X_{t}}\left(\zeta_{+}^{X}<\infty\right)\right)=\mathbb{E}_{\delta_{x}}\left(\mathrm{e}^{-\left\langle\Phi, X_{t}^{+}\right\rangle}\right)=\mathbb{E}_{\delta_{x}}\left(\mathrm{e}^{-\left\langle\Phi, X_{t}\right\rangle}\right) \quad \text { for all } x, t \geq 0
$$

Inspecting the semigroup evolution equation (4) for $X$ with data $f=\Phi$ and taking account of the fact that its unique solution is given by (3), we see that $\Phi$ solves the differential equation in (5).

To show that $\Phi(+\infty)=\lambda^{*}$, note that the law of $\left\langle\Phi, X_{t}^{+}\right\rangle$under $\mathbb{P}_{\delta_{x}}$ is equal to that of $\left\langle\Phi(x+\cdot),\left.Y_{D_{-x}^{t}}\right|_{(-x, \infty) \times\{t\}}\right\rangle$ under $\mathbb{Q}_{\delta_{0}}$. Due to the monotonicity of $\Phi(x)$ and $\left.Y_{D_{-x}^{t}}\right|_{(-x, \infty) \times\{t\}}$ in $x$ and the fact that $0<\Phi(x) \leq \lambda^{*}$ we have, with the help of dominated convergence,

$$
\mathrm{e}^{-\Phi(+\infty)}=\lim _{x \uparrow \infty} \mathbb{Q}_{\delta_{0}}\left(\exp \left\{-\left\langle\Phi(x+\cdot),\left.Y_{D_{-x}^{t}}\right|_{(-x, \infty) \times\{t\}}\right\rangle\right)=\mathbb{Q}_{\delta_{0}}\left(\mathrm{e}^{-\Phi(\infty)\left\|Y_{t}\right\|}\right) .\right.
$$

On account of the fact that the process $\left\{\left\|Y_{t}\right\|: t \geq 0\right\}$ is a continuous-state branching process with branching mechanism $\psi$, the equality in (11) together with the fact that $\Phi(+\infty) \in\left(0, \lambda^{*}\right]$ forces us to deduce that $\Phi(+\infty)=\lambda^{*}$. 
Now suppose that $\phi$ solves (5). The backbone decomposition tells us that, for all $t \geq 0$, $Z_{t}(\cdot)$ given $\Lambda_{t}^{X}(\cdot)$ is a Poisson random field with intensity measure $\lambda^{*} \Lambda_{t}^{X}(\cdot)$. Hence,

$$
\begin{aligned}
\mathbf{E}_{\delta_{x}}\left[\mathrm{e}^{\left\langle\log \left(1-\phi / \lambda^{*}\right), Z_{t}^{X}\right\rangle}\right] & =\mathbf{E}_{\delta_{x}} \mathbf{E}\left[\mathrm{e}^{\left\langle\log \left(1-\phi / \lambda^{*}\right), Z_{t}^{X}\right\rangle} \mid \Lambda_{t}^{X}\right] \\
& =\mathbf{E}_{\delta_{x}}\left[\exp \left\{-\int\left(1-\mathrm{e}^{\log \left(1-\phi(y) / \lambda^{*}\right)}\right) \lambda^{*} \Lambda_{t}^{X}(\mathrm{~d} y)\right\}\right] \\
& =\mathbf{E}_{\delta_{x}}\left[\mathrm{e}^{-\left\langle\phi, \Lambda_{t}^{X}\right\rangle}\right] \\
& =\mathrm{e}^{-\phi(x)}
\end{aligned}
$$

Recalling that $\phi$ is monotone with $\phi(0+)=0$ and $\phi(+\infty)=\lambda^{*}$, and, hence, that $-\log (1-$ $\left.\phi / \lambda^{*}\right) \in[0, \infty)$ so that

$$
\left\langle-\log \left(1-\frac{\phi}{\lambda^{*}}\right), Z_{t}^{X}\right\rangle \geq-\log \left(1-\phi\left(R_{t}^{Z^{X}}\right)\right),
$$

it follows with the help of the known asymptotics of $R_{t}^{Z^{X}}$, e.g. (10), that

$$
\begin{aligned}
& \limsup _{t \rightarrow \infty} \mathbf{E}_{\delta_{x}}\left[\mathrm{e}^{\left\langle\log \left(1-\phi / \lambda^{*}\right), Z_{t}^{X}\right\rangle} \mathbf{1}_{\left\{\zeta_{+}^{Z^{X}}=\infty\right\}}\right] \\
& \quad \leq \limsup _{t \rightarrow \infty} \mathbf{E}_{\delta_{x}}\left[\mathrm{e}^{\log \left(1-\phi\left(R_{t}^{Z^{X}}\right) / \lambda^{*}\right)} \mathbf{1}_{\left\{\zeta_{+}^{Z^{X}}=\infty\right\}}\right] \\
& \quad=0 .
\end{aligned}
$$

Subsequently,

$$
\begin{aligned}
\mathrm{e}^{-\phi(x)} & =\mathbf{P}_{\delta_{x}}\left(\zeta_{+}^{Z^{X}}<\infty\right)+\lim _{t \rightarrow \infty} \mathbf{E}_{\delta_{x}}\left[\mathrm{e}^{-\left\langle-\log \left(1-\phi / \lambda^{*}\right), Z_{t}^{X}\right\rangle} \mathbf{1}_{\left\{\zeta_{+}^{Z^{X}}=\infty\right\}}\right] \\
& =\mathbf{P}_{\delta_{x}}\left(\zeta_{+}^{Z^{X}}<\infty\right) .
\end{aligned}
$$

We conclude from (12) that

$$
\Phi(x)=-\log \mathbf{P}_{\delta_{x}}\left(\zeta_{+}^{\Lambda^{X}}<\infty\right)=-\log \mathbf{P}_{\delta_{x}}\left(\zeta_{+}^{Z^{X}}<\infty\right)
$$

is the unique monotone solution to (5). Moreover, since $\left\{\zeta_{+}^{\Lambda^{X}}<\infty\right\} \subseteq\left\{\zeta_{+}^{Z^{X}}<\infty\right\}$, we may now also deduce that $\left\{\zeta_{+}^{\Lambda^{X}}<\infty\right\}=\left\{\zeta_{+}^{Z^{X}}<\infty\right\}, \mathbf{P}_{\delta_{x}}$-almost surely, which completes the proof of part (i) of the theorem.

Proof of (ii). Suppose now that $\rho \geq \sqrt{2 \alpha}$. The estimate $R_{t}^{X} \leq R_{t}^{Y}$ used in (9) now tells us that $\mathbb{P}_{\delta_{x}}\left(\zeta_{+}^{X}<\infty\right)=1$ and, hence, because of the backbone decomposition, it also tells us that $\mathbf{P}_{\delta_{x}}\left(\zeta_{+}^{\Lambda^{X}}<\infty\right)=1$. As noted earlier, the Poisson embedding of $Z^{X}$ in $\Lambda^{X}$ gives us that $\left\{\zeta_{+}^{\Lambda^{X}}<\infty\right\} \subseteq\left\{\zeta_{+}^{Z^{X}}<\infty\right\}$ and, hence, it follows that $\mathbf{P}_{\delta_{x}}\left(\zeta_{+}^{Z^{X}}<\infty\right)=1$. Suppose now that a monotone wave solution, $\Phi$, to (5) exists. Then the computation in (12) forces us to conclude that $\Phi \equiv 0$, which is a contradiction. Therefore, there can be no solutions to (5).

Remark 2. Whilst Theorem 1 offers results on the existence and uniqueness of solutions to (5), Proposition 2 of [22] and Theorem 1 of [15] also offer the rate of decay of monotone solutions at $+\infty$ to the wave equation

$$
\frac{1}{2} \Psi^{\prime \prime}(x)-\rho \Psi^{\prime}(x)+F(\Psi(x)) \quad \text { on } x>0 \text { with } \Psi(0+)=1 \text { and } \Psi(+\infty)=0
$$

for $\rho<\sqrt{2 q}$, where $F(s)=q\left(s^{2}-s\right)$ and $q>0$. A straightforward inspection of the proof of Theorem 1 of [15] shows that in fact their result on the decay of $\Psi$ holds for more general 
functions $F$ taking, for example, the form $F(s)=q\left(\sum_{n=2}^{\infty} s^{n} p_{n}-s\right)$ for $s \in[0,1]$ and $q>0$, where $\left\{p_{n}: n \geq 2\right\}$ is a probability distribution satisfying $F^{\prime}(1)<\infty$. Specifically, most of the arguments in [15] do not require a dyadic offspring distribution such as is imposed there; however, in [15, Section 6] one must take care with the exponential term in the martingale defined in Equation (14). In their terminology, the integrand in the exponential term, $\beta\left(f\left(Y_{S}\right)-1\right)$, needs to be replaced by $G\left(Y_{s}\right)$, where $G(s)=F(s) / s$. Thereafter, the necessary adjustments, which pertain largely to bounds, are relatively obvious. In that case their result reads as follows. For all $\rho<\sqrt{2 F^{\prime}(1)}$

$$
\lim _{x \uparrow \infty} \exp \left\{-\left(\rho-\sqrt{\rho^{2}+2 q}\right) x\right\} \Psi(x)=k_{\rho}
$$

for some $k_{\rho} \in(0, \infty)$.

Note that, when $F$ is given by (8), it is straightforward to check that $\Psi$ solves (13) if and only if $\lambda^{*}(1-\Psi)$ solves (5). It follows immediately that, when $\rho<\sqrt{2 \alpha}$,

$$
\lim _{x \uparrow \infty} \exp \left\{-\left(\rho-\sqrt{\rho^{2}+2 \psi^{\prime}\left(\lambda^{*}\right)}\right) x\right\}\left(1-\frac{\Phi(x)}{\lambda^{*}}\right)=k_{\rho} .
$$

\section{Proof of Theorem 2}

As alluded to above, our objective is to embed an existing result for branching Brownian motion with absorption at the origin into the superprocess setting with the help of the backbone decomposition. For all $x \in \mathbb{R}$, we shall denote by $\mathrm{Q}_{\delta_{x}}$ the law of the backbone decomposition, $\left(Z^{Y}, \Lambda^{Y}\right)$ of $Y$. The existing result in question is due to Maillard [20] and when paraphrased in terms of the backbone process $Z^{Y}$ for $Y$, states that, under the condition that $\sum_{n \geq 2} n(\log n)^{2+\varepsilon} p_{n}<\infty$ for some $\varepsilon>0$, and $\rho=\sqrt{2 \alpha}$, it follows that, for all $x \geq-z$,

$$
\mathrm{Q}_{\delta_{x}}\left(\left\|Z_{D_{-z}}^{Y}\right\|>n \mid\left\|Z_{0}^{Y}\right\|=1\right) \sim \frac{\sqrt{2 \alpha}(x+z) \mathrm{e}^{\sqrt{2 \alpha}(x+z)}}{n(\log n)^{2}}
$$

as $\mathbb{N} \ni n \uparrow \infty$. Here we understand $Z_{D_{-z}}^{Y}$ to mean the atomic-valued measure, supported on $\{-z\} \times[0, \infty)$, which describes the space-time positions of particles in the branching diffusion $Z^{Y}$ that are first in their line of descent to exit the domain $D_{-z}^{\infty}$. The process $\left\|Z_{D}^{Y}\right\|:=\left\{\left\|Z_{D_{-z}}^{Y}\right\|: z \geq 0\right\}$ is known to be a continuous-time Galton-Watson (cf. Lemma 3.1 and Proposition 3.2 of [20] or Proposition 3 of [21]), which, like its continuous-state space analogue $\left\|Y_{D}\right\|$, has growth rate $\sqrt{2 \alpha}$. Maillard's result follows by first establishing that

$$
F_{z}(s):=\mathrm{Q}_{\delta_{0}}\left(s^{\left\|Z_{D_{-z}}^{Y}\right\|} \mid\left\|Z_{0}^{Y}\right\|=1\right)
$$

satisfies

$$
F_{z}^{\prime \prime}(1-s) \sim \frac{\sqrt{2 \alpha} z \mathrm{e}^{z \sqrt{2 \alpha}}}{s(\log (1 / s))^{2}} \quad \text { as } s \downarrow 0,
$$

and then applying a classical Tauberian theorem.

The strategy for our proof of Theorem 2 will be to first show that the moment condition $\sum_{n \geq 2} n(\log n)^{2+\epsilon} p_{n}<\infty$ is implied by (7). Thereafter, we shall appeal to an analytical identity that arises through the Poissonisation property of the backbone decomposition, thereby allowing us to convert the asymptotic (16) into an appropriate asymptotic which leads, again through an application of a Tauberian theorem, to the conclusion of Theorem 2. We start with the following lemma. 
Lemma 1. If $\int_{[1, \infty)} x(\log x)^{2+\varepsilon} \Pi(\mathrm{d} x)<\infty$ for some $\varepsilon>0$ then $\sum_{n \geq 2} n(\log n)^{2+\varepsilon} p_{n}<\infty$.

Proof. Appealing to the definition of $\left\{p_{n}: n \geq 2\right\}$, it suffices to prove that

$$
\int_{0}^{\infty} \sum_{n \geq 2} n(\log n)^{2+\varepsilon} \frac{\left(\lambda^{*} x\right)^{n}}{n !} \mathrm{e}^{-\lambda^{*} x} \Pi(\mathrm{d} x)<\infty .
$$

To this end, define the function $f(x)=(\log (1+x))^{2+\varepsilon}$. Then it is easy to see that

$$
f^{\prime \prime}(x)=-(2+\varepsilon) \frac{(\log (1+x))^{\varepsilon}}{(1+x)^{2}}(\log (1+x)-(1+\varepsilon)) .
$$

We can find $N_{0} \in \mathbb{N}$ such that $\log \left(1+N_{0}\right)>(1+\varepsilon)$ and, subsequently, that $f^{\prime \prime}(x)<0$ for $x \geq N_{0}$. This implies that $f$ is concave in $\left(N_{0}, \infty\right)$. Hence, using Jensen's inequality,

$$
\begin{array}{rl}
\sum_{n \geq N_{0}+1} & n(\log n)^{2+\varepsilon} \frac{\left(\lambda^{*} x\right)^{n}}{n !} \mathrm{e}^{-\lambda^{*} x} \\
\quad= & \lambda^{*} x \sum_{n \geq N_{0}}(\log (n+1))^{2+\varepsilon} \frac{\left(\lambda^{*} x\right)^{n}}{n !} \mathrm{e}^{-\lambda^{*} x} \\
\leq & \left(\lambda^{*} x\right)\left(\sum_{n \geq N_{0}} \mathrm{e}^{-\lambda^{*} x} \frac{\left(\lambda^{*} x\right)^{n}}{n !}\right)\left(\log \left(\frac{\sum_{n \geq N_{0}} n\left(\lambda^{*} x\right)^{n} \mathrm{e}^{-\lambda^{*} x} / n !}{\sum_{n \geq N_{0}}\left(\lambda^{*} x\right)^{n} \mathrm{e}^{-\lambda^{*} x} / n !}+1\right)\right)^{2+\varepsilon} \\
\leq & \left(\lambda^{*} x\right)\left(\log \left(\frac{\sum_{n \geq N_{0}} n\left(\lambda^{*} x\right)^{n} / n !}{\sum_{n \geq N_{0}}\left(\lambda^{*} x\right)^{n} / n !}+1\right)\right)^{2+\varepsilon} .
\end{array}
$$

On the other hand, we have

$$
\lim _{x \rightarrow \infty} \log \left(\frac{\sum_{n \geq N_{0}} n\left(\lambda^{*} x\right)^{n} / n !}{\sum_{n \geq N_{0}}\left(\lambda^{*} x\right)^{n} / n !}+1\right) / \log x=1 .
$$

So we can find $K>0$ such that, if $x>K$,

$$
\log \left(\frac{\sum_{n \geq N_{0}} n\left(\lambda^{*} x\right)^{n} / n !}{\sum_{n \geq N_{0}}\left(\lambda^{*} x\right)^{n} / n !}+1\right)<2 \log x .
$$

Using (18), this implies that

$$
\int_{[K, \infty)} \sum_{n \geq N_{0}+1} n(\log n)^{2+\varepsilon} \frac{\left(\lambda^{*} x\right)^{n}}{n !} \mathrm{e}^{-\lambda^{*} x} \Pi(\mathrm{d} x)<2^{2+\varepsilon} \lambda^{*} \int_{[K, \infty)} x(\log x)^{2+\varepsilon} \Pi(\mathrm{d} x)<\infty .
$$

On the other hand, by choosing $N_{0}$ large enough, we also have, for all $n \geq N_{0}+1,(\log n)^{2+\varepsilon}<$ $C(n-1)$ for some $C>0$. Hence,

$$
\begin{aligned}
\int_{(0, K)} & \sum_{n \geq N_{0}+1} n(\log n)^{2+\varepsilon} \frac{\left(\lambda^{*} x\right)^{n}}{n !} \mathrm{e}^{-\lambda^{*} x} \Pi(\mathrm{d} x) \\
\leq & \left(\lambda^{*} K\right)^{2} \sum_{n \geq N_{0}+1} \int_{(0, \infty)} \frac{\left(\lambda^{*} x\right)^{n-2}}{(n-2) !} \mathrm{e}^{-\lambda^{*} x} \Pi(\mathrm{d} x) \\
\leq & \left(\lambda^{*} K\right)^{2} \sum_{n \geq 2} p_{n}<\infty .
\end{aligned}
$$


For the first terms of (17), we have

$$
\begin{aligned}
\int_{(0, \infty)} \sum_{n=2}^{N_{0}} n(\log n)^{2+\varepsilon} \frac{\left(\lambda^{*} x\right)^{n}}{n !} \mathrm{e}^{-\lambda^{*} x} \Pi(\mathrm{d} x) & =\sum_{n=2}^{N_{0}} n(\log n)^{2+\varepsilon} \frac{\left(\lambda^{*}\right)^{n}}{n !} \int_{(0, \infty)} x^{n} \mathrm{e}^{-\lambda^{*} x} \Pi(\mathrm{d} x) \\
& <\infty,
\end{aligned}
$$

which follows from the fact that each term of the sum is finite. This completes the proof.

Remark 3. It is not difficult to show that the converse of the statement in Lemma 1 is also true; however, we leave it as an exercise for the reader.

Let us now turn to the proof of Theorem 2. We approach the proof here on in two steps. The first step is to show that the process $\left\|Z_{D}^{Y}\right\|$ under $\mathrm{Q}_{\delta_{x}}$, for which distributional properties are known thanks to (14), has the same branching generator as the continuous-time Galton-Watson process $Z^{\prime}:=\left\{Z_{z}^{\prime}: z \geq 0\right\}$, where the latter is the backbone embedded in the continuous-state branching process $\left\|Y_{D}\right\|$. Thanks to the backbone decomposition of $\left\|Y_{D}\right\|$, say $\left(Z^{\prime}, \Lambda^{\prime}\right)$, and the easily seen fact that $\psi_{D}\left(\lambda^{*}\right)=0$, the law of $Z_{z}^{\prime}$ given $\Lambda_{z}^{\prime}$ is that of a Poisson random variable with parameter $\lambda^{*} \Lambda_{z}^{\prime}$. This Poissonisation result will allow us to feed the known distributional asymptotic for $Z_{z}^{\prime}$ (equivalently, $\left\|Z_{D_{-z}}^{Y}\right\|$ ) into the required result for $\Lambda_{z}^{\prime}$ (equivalently, $\left\|Y_{D_{-z}}\right\|$ ).

Step 1. We start by recalling from [20, Section 3] that $\left\|Z_{D}^{Y}\right\|$ has branching generator given by $\Theta^{\prime}\left(\Theta^{-1}(s)\right), s \in[0,1]$, where $\Theta$ is the unique monotone solution to the wave equation

$$
\frac{1}{2} \Theta^{\prime \prime}(x)-\sqrt{2 \alpha} \Theta^{\prime}(x)+F(\Theta(x))=0 \quad \text { on } \mathbb{R} \text { with } \Theta(-\infty)=1 \text { and } \Theta(+\infty)=0,
$$

and $F$ is the branching generator of the backbone $Z^{Y}$ given in (8). Again, appealing to (8) but for the backbone decomposition $\left(Z^{\prime}, \Lambda^{\prime}\right)$ of $\left\|Y_{D}\right\|$ and the fact that $\psi_{D}\left(\lambda^{*}\right)=0$, we know that $Z^{\prime}$ has branching generator given by

$$
F_{D}(s)=\frac{1}{\lambda^{*}} \psi_{D}\left(\lambda^{*}(1-s)\right) \text { for } s \in[0,1] .
$$

Our objective is thus to show that $F_{D}(s)=\Theta^{\prime}\left(\Theta^{-1}(s)\right)$ for all $s \in[0,1]$.

To this end, recall that $\psi_{D}(\lambda)=\Psi^{\prime}\left(\Psi^{-1}(\lambda)\right)$ for $\lambda \in\left[0, \lambda^{*}\right]$, where $\Psi$ solves (6). It is a straightforward exercise to check that $\Theta(x)=1-\Psi(-x) / \lambda^{*}$. Indeed, $\Theta(+\infty)=0$ and $\Theta(-\infty)=1$, and $\Theta$ solves (19) on account of the fact that $\Psi$ solves (6). Moreover, we readily confirm that

$$
\frac{1}{\lambda^{*}} \Psi^{\prime}\left(\Psi^{-1}\left(\lambda^{*}(1-s)\right)\right)=\Theta^{\prime}\left(\Theta^{-1}(s)\right) \text { for } s \in[0,1] .
$$

This implies in turn that the required equality, $F_{D}(s)=\Theta^{\prime}\left(\Theta^{-1}(s)\right)$, holds, and in particular that $\left\|Z_{D}^{Y}\right\|$ and $Z^{\prime}$ have the same branching generator.

Step 2. Recall that $\left(Z^{\prime}, \Lambda^{\prime}\right)$ is the backbone decomposition of $\left\{\left\|Y_{D_{-z}}\right\|: z \geq 0\right\}$, and denote the law of the former by $\mathcal{Q}_{x}$ when the latter has law $\mathbb{Q}_{\delta_{x}}$. Appealing to spatial homogeneity, we may henceforth proceed without loss of generality by assuming that $x=0$.

It follows from the conclusion of step 1 and the Poissonisation property of the backbone decomposition that, for $z \geq 0$ and $s \in[0,1]$,

$$
\begin{aligned}
\mathrm{Q}_{\delta_{0}}\left(s\left\|Z_{D_{-z}}^{Y}\right\|\right. & =\mathcal{Q}_{0}\left(s^{Z_{z}^{\prime}}\right) \\
& =\mathcal{Q}_{0}\left(\mathcal{Q}_{0}\left(s^{\prime} \mid \Lambda_{z}^{\prime}\right)\right) \\
& =\mathcal{Q}_{0}\left(\mathrm{e}^{-\lambda^{*} \Lambda_{z}^{\prime}(1-s)}\right) \\
& =\mathbb{Q}_{\delta_{0}}\left(\exp \left\{-\lambda^{*}\left\|Y_{D_{-z}}\right\|(1-s)\right\}\right) .
\end{aligned}
$$


Now, using the fact that, under $\mathrm{Q}_{\delta_{0}},\left\|Z_{D_{0}}^{Y}\right\|=\left\|Z_{0}^{Y}\right\|$ is a Poisson random variable with intensity $\lambda^{*}$, we have

$$
\mathrm{Q}_{\delta_{0}}\left[s^{\left\|Z_{D_{-}}^{Y}\right\|}\right]=\sum_{k=0}^{\infty} \mathrm{e}^{-\lambda^{*}} \frac{\left(\lambda^{*}\right)^{k}}{k !} F_{z}(s)^{k}=\exp \left\{-\lambda^{*}\left(1-F_{z}(s)\right)\right\}
$$

where $F_{z}(s)$ was defined in (15). If we set

$$
w_{z}(s)=\mathbb{Q}_{\delta_{0}}\left(\mathrm{e}^{-s\left\|Y_{D_{-z}}\right\|}\right)
$$

then (20) and (21) tell us that

$$
w_{z}\left(\lambda^{*} s\right)=\exp \left\{-\lambda^{*}\left(1-F_{z}(1-s)\right)\right\}
$$

Taking second derivatives on both sides of the above equality gives

$$
\left(\lambda^{*}\right)^{2} w_{z}^{\prime \prime}\left(\lambda^{*} s\right)=\left(\lambda^{*} F_{z}^{\prime \prime}(1-s)+\left(\lambda^{*} F_{z}^{\prime}(1-s)\right)^{2}\right) \exp \left\{-\lambda^{*}\left(1-F_{z}(1-s)\right)\right\} .
$$

Recalling (16) and noting that

$$
F_{z}^{\prime}(1-s) \sim \mathrm{e}^{z \sqrt{2 \alpha}} \quad \text { as } s \downarrow 0
$$

which holds on account of the fact that $\left\|Z_{D}^{Y}\right\|$ is a continuous-time Galton-Watson process with growth rate $\sqrt{2 \alpha}$, we have, from (22),

$$
w_{z}^{\prime \prime}\left(\lambda^{*} s\right) \sim \frac{\sqrt{2 \alpha} z \mathrm{e}^{z \sqrt{2 \alpha}}}{\lambda^{*} s(\log (1 / s))^{2}} \quad \text { as } s \downarrow 0 .
$$

Taking $u=\lambda^{*} s$ and using (23) we obtain

$$
w_{z}^{\prime \prime}(u) \sim \frac{\sqrt{2 \alpha} z \mathrm{e}^{z \sqrt{2 \alpha}}}{u\left(\log \lambda^{*}+\log (1 / u)\right)^{2}} \sim \frac{\sqrt{2 \alpha} z \mathrm{e}^{z \sqrt{2 \alpha}}}{u(\log (1 / u))^{2}} \quad \text { as } u \downarrow 0 .
$$

Denote by $U_{z}(\mathrm{~d} y)$ the measure in $(0, \infty)$ defined by the relation

$$
w_{z}(s)=\int_{[0, \infty)} \mathrm{e}^{-s y} U_{z}(\mathrm{~d} y) .
$$

In other words, $U_{z}(\mathrm{~d} y)=\mathbb{Q}_{\delta_{0}}\left(\left\|Y_{D_{-z}}\right\| \in \mathrm{d} y\right)$ for $y \geq 0$. Let us take $\tilde{U}_{z}(\mathrm{~d} y)=y^{2} U_{z}(\mathrm{~d} y)$ on $[0, \infty)$. Then it is easy to see that

$$
w_{z}^{\prime \prime}(s)=\int_{[0, \infty)} \mathrm{e}^{-s y} \tilde{U}_{z}(\mathrm{~d} y)
$$

Using Theorem 2 XIII.5 of [13], we have, using (24),

$$
\tilde{U}_{z}(t) \sim \frac{t \sqrt{2 \alpha} z \mathrm{e}^{z \sqrt{2 \alpha}}}{(\log t)^{2}} \text { as } t \rightarrow \infty .
$$


Using integration by parts, it is easy to see that

$$
\begin{aligned}
U_{z}(t, \infty) & =\int_{(t, \infty)} y^{-2} \tilde{U}_{z}(\mathrm{~d} y) \\
& \sim \sqrt{2 \alpha} z \mathrm{e}^{z \sqrt{2 \alpha}}\left(2 \int_{t}^{\infty} \frac{1}{y^{2}(\log y)^{2}} \mathrm{~d} y-\frac{1}{t(\log t)^{2}}\right) \quad \text { as } t \rightarrow \infty
\end{aligned}
$$

But, by Theorem 1 VIII.9 of [13], the integral on the right-hand side above is equivalent to $1 / t(\log t)^{2}$. This implies that

$$
\mathbb{Q}_{\delta_{0}}\left(\left\|Y_{D_{-z}}\right\|>t\right)=U_{z}(t, \infty) \sim \frac{\sqrt{2 \alpha} z \mathrm{e}^{z \sqrt{2 \alpha}}}{t(\log t)^{2}} \quad \text { as } t \rightarrow \infty,
$$

which proves the result.

Remark 4. Maillard [20] gave further results in the case that $\rho>\sqrt{2 \alpha}$ for the asymptotic behaviour of $\mathbb{Q}_{\delta_{x}}\left(\left\|Y_{D_{-z}}\right\|>t\right)$ as $t \uparrow \infty$. Again, using ideas of Poisson embedding through the backbone, analogous asymptotics can be transferred from the case of branching Brownian motion to super-Brownian motion.

\section{Acknowledgements}

All three authors would like to thank an anonymous referee for their comments and remarks on an earlier draft of this paper which lead to its improvement. AEK acknowledges financial support from the Santander Research Grant Fund, JLP acknowledges financial support from CONACyT-MEXICO, grant number 150645, and AMS acknowledges financial support from CONACyT-MEXICO, grant number 129076.

\section{Added in proof}

Since the appearance of Yang and Ren [28], the moment condition in Maillard [20] can be weakened so that, in the current context, $\sum_{n \geq 2} n(\log n)^{2+\varepsilon} p_{n}<\infty$ can be replaced by $\sum_{n \geq 2} n(\log n)^{2} p_{n}<\infty$. Accordingly, it is trivial to make the adjustments in the given arguments to weaken the assumption in Theorem 2 from $\int_{[1, \infty)} x(\log x)^{2+\varepsilon} \Pi(\mathrm{d} x)<\infty$ to $\int_{[1, \infty)} x(\log x)^{2} \Pi(\mathrm{d} x)<\infty$.

\section{References}

[1] Addario-Berry, L. and Broutin, N. (2011). Total progeny in killed branching random walk. Prob. Theory Relat. Fields 151, 265-295.

[2] AḮÉKon, E., Hu, Y. AND ZINDY, O. (2011). The precise tail behavior of the total progeny of a killed branching random walk. Preprint. Available at http://arxiv.org/abs/1102.5536v1.

[3] Berestycki, J., Kyprianou, A. E. and Murillo-Salas, A. (2011). The prolific backbone decomposition for supercritical superdiffusions. Stoch. Process. Appl. 121, 1315-1331.

[4] Dynkin, E. B. (1991). Branching particle systems and superprocesses. Ann. Prob. 19, 1157-1194.

[5] Dynkin, E. B. (1991). A probabilistic approach to one class of nonlinear differential equations. Prob. Theory Relat. Fields 89, 89-115.

[6] Dynkin, E. B. (1993). Superprocesses and partial differential equations. Ann. Prob. 21, 1185-1262.

[7] Dynkin, E. B. (2001). Branching exit Markov systems and superprocess. Ann. Prob. 29, 1833-1858.

[8] Dynkin, E. B. (2002). Diffusions, Superdiffusions and Partial Differential Equations. American Mathematical Society, Providence, RI.

[9] Dynkin, E. B. And Kuznetsov, S. E. (2004). N-measures for branching exit Markov systems and their applications to differential equations. Prob. Theory Relat. Fields 130, 135-150. 
[10] El Karoui, N. and Roelly, S. (1991). Propriétés de martingales, explosion et représentation de LévyKhintchine d'une classe de processus de branchement à valeurs mesures. Stoch. Process. Appl. 38, 239-266.

[11] Engländer, J. AND Pinsky, R. G. (1999). On the construction and support properties of measure-valued diffusions on $D \subseteq \mathbb{R}^{d}$ with spatially dependent branching. Ann. Prob. 27, 684-730.

[12] Evans, S. N. AND O'Connell, N. (1994). Weighted occupation time for branching particle systems and a representation for the supercritical superprocess. Canad. Math. Bull. 37, 187-196.

[13] Feller, W. (1971). An Introduction to Probability Theory and Its Applications, Vol. II, 2nd edn. John Wiley, New York.

[14] Fitzsimmons, P. J. (1988). Construction and regularity of measure-valued Markov branching processes. Israel J. Math. 64, 337-361.

[15] Harris, J. W., Harris, S. C. and Kyprianou, A. E. (2006). Further probabilistic analysis of the FisherKolmogorov-Pretrovskii-Piscounov equation: one sided travelling-waves. Ann. Inst. H. Poincaré Prob. Statist. 42, 125-145.

[16] KametaKa, Y. (1976). On the nonlinear diffusion equation of Kolmogorov-Petrovskii-Piskunov type. Osaka J. Math. 13, 11-66.

[17] Kyprianou, A. E. (2006). Introductory Lectures on Fluctuations of Lévy Processes with Applications. Springer, Berlin.

[18] Kyprianou, A., Liu, R.-L., Murillo-Salas, A. And Ren, Y.-X. (2012). Supercritical super-Brownian motion with a general branching mechanism and travelling waves. Ann. Inst. H. Poincaré Prob. Statist. 48, 661-687.

[19] Le Gall, J.-F. (1999). Spatial Branching Processes, Random Snakes and Partial Differential Equations. Birkhäuser, Basel.

[20] MaillaRd, P. (2012). The number of absorbed individuals in branching Brownian motion with a barrier. To appear in Ann. Inst. H. Poincaré Prob. Statist. .

[21] Neveu, J. (1988). Multiplicative martingales for spatial branching processes. In Seminar on Stochastic Processes (Prog. Prob. Statist. 15), eds E. Çinlar, K. L. Chung and R. K. Getoor, Birkhaüser, Boston, MA, pp. $223-241$.

[22] PINSKY, R. G. (1995). K-P-P-type asymptotics for nonlinear diffusion in a large ball with infinite boundary data and on $\mathbf{R}^{d}$ with infinite initial data outside a large ball. Commun. Partial Differential Equat. 20, 1369-1393.

[23] Salisbury, T. and Verzani, J. (1999). On the conditioned exit measures of super Brownian motion. Prob. Theory Relat. Fields 115, 237-285.

[24] Salisbury, T. S. AND Verzani, J. (2000). Non-degenerate conditionings of the exit measure of super Brownian motion. Stoch. Process. Appl. 87, 25-52.

[25] SHeU, Y.-C. (1997). Lifetime and compactness of range for super-Brownian motion with a general branching mechanism. Stoch. Process. Appl. 70, 129-141.

[26] Uсhiуama, K. (1978). The behavior of solutions of some non-linear diffusion equations for large time. J. Math. Kyoto Univ. 18, 453-508.

[27] Watanabe, K. (1968). A limit theorem of branching processes and continuous state branching processes. J. Math. Kyoto Univ. 8, 141-167.

[28] YANG, T. AND REN, Y.-X. (2011). Limit theorem for derivative martingale at criticality w.r.t. branching Brownian motion. Statist. Prob. Lett. 81, 195-200. 